\title{
Hospital discharges-based search of acute flaccid paralysis cases 2007-2016 in Italy and comparison with the National Surveillance System for monitoring the risk of polio reintroduction
}

Paola Stefanelli ${ }^{*}$, Stefania Bellino ${ }^{\dagger}$ (D) Stefano Fiore, Stefano Fontana, Concetta Amato, Gabriele Buttinelli and the Regional Reference Centres of the National Surveillance System for Acute flaccid paralysis

\begin{abstract}
Background: Acute flaccid paralysis (AFP) surveillance has been adopted globally as a key strategy for monitoring the progress of the polio eradication initiative. Hereby, to evaluate the completeness of the ascertainment of AFP cases in Italy, a hospital-discharges based search was carried out.

Methods: AFP cases occurring between 2007 and 2016 among children under 15 years of age were searched in the Italian Hospital Discharge Records (HDR) database using specific ICD-9-CM diagnostic codes. AFP cases identified between 2015 and 2016 were then compared with those notified to the National Surveillance System (NSS).

Results: Over a 10-year period, 4163 hospital discharges with diagnosis of AFP were reported in Italy. Among these, 956 (23.0\%) were acute infective polyneuritis, 1803 (43.3\%) myopathy, and 1408 (33.8\%) encephalitis, myelitis and encephalomyelitis. During the study period, a decreasing trend was observed for all diagnoses and overall the annual incidence rate (IR) declined from 5.5 to 4.5 per 100,000 children. Comparing NSS with HDR data in 2015-2016, we found a remarkable underreporting, being AFP cases from NSS only 14\% of those recorded in HDR. In particular, the acute infective polyneuritis cases reported to NSS accounted for $42.6 \%$ of those detected in HDR, while only $0.9 \%$ of myopathy cases and $13.1 \%$ of encephalitis/myelitis/encephalomyelitis cases have been notified to NSS. The highest AFP IRs per 100,000 children calculated on HDR data were identified in Liguria (17.4), Sicily (5.7), and Veneto (5.1) Regions; regarding the AFP notified to the NSS, 11 out of 21 Regions failed to reach the number of expected cases (based on 1/100,000 rate), and the highest discrepancies were observed in the Northern Regions. Overall, the national AFP rate was equal to 0.6 , therefore did not reach the target value.
\end{abstract}

Conclusions: AFP surveillance data are the final measure of a country's progress towards polio eradication. The historical data obtained by the HDR have been useful to assess the completeness of the notification data and to identify the Regions with a low AFP ascertainment rate in order to improve the national surveillance system.

Keywords: Acute flaccid paralysis, Hospital discharge records, National surveillance system, Polio

\footnotetext{
* Correspondence: paola.stefanelli@iss.it

${ }^{\dagger}$ Paola Stefanelli and Stefania Bellino contributed equally to this work.

Department of Infectious Diseases, Italian National Institute of Health (Istituto

Superiore di Sanità), Rome, Italy
}

(c) The Author(s). 2019 Open Access This article is distributed under the terms of the Creative Commons Attribution 4.0 International License (http://creativecommons.org/licenses/by/4.0/), which permits unrestricted use, distribution, and reproduction in any medium, provided you give appropriate credit to the original author(s) and the source, provide a link to the Creative Commons license, and indicate if changes were made. The Creative Commons Public Domain Dedication waiver (http://creativecommons.org/publicdomain/zero/1.0/) applies to the data made available in this article, unless otherwise stated. 


\section{Background}

The World Health Organization (WHO) recommends countries to monitor cases of acute flaccid paralysis (AFP), which is the main clinical picture of a poliovirus infection, and the poliovirus (PV) circulation, to provide accurate data for the development of appropriate prevention and control strategies [1]. An important prerequisite for poliofree certification is that the National Surveillance System (NSS) successfully detects annually at least one case of non-polio associated AFP per 100,000 children under 15 years of age and no cases of wild polio occur for three consecutive years $[1,2]$. The adequacy and quality of the AFP surveillance are assessed by the following key indicators recommended by the WHO: completeness of reporting, geographical and demographic representativeness of reporting sites, sensitivity of surveillance, completeness of case investigations and follow-up, laboratory performance [1]. Although low levels of PV transmission have been reported in recent years, the virus is still endemic in Nigeria, Afghanistan, and Pakistan. Until the PV transmission is not interrupted, all countries remain at risk of virus importation, through population movements. Indeed, polio eradication is hampered by the international spread of poliovirus through travellers. In response to the ongoing importations of poliovirus in polio-free countries, the WHO Director General on 5th May 2014 declared the international spread of wild poliovirus to be a public health emergency of international concern [3].

In Italy, AFP surveillance was established in 1994, according to the WHO guidelines, but started in January 1996 as a pilot study on four Italian Regions, and was finally extended to the whole nation in 1997. The Italian National Institute of Health (Istituto Superiore di Sanità - ISS) and the Ministry of Health $(\mathrm{MoH})$ coordinate the national AFP surveillance system including 20 Regional Reference Centres (RRC).

Acute flaccid paralysis is defined as any case of new onset of hypotonic weakness in a child younger than 15 years of age and includes possible diseases due to paralytic poliomyelitis, Guillain-Barré Syndrome (GBS), transverse myelitis, polyradiculoneuritis, traumatic and neoplastic neuritis [4].

The present study aims to investigate the number of AFP cases reported from 2007 to 2016 in the Hospital Discharge Records (HDRs). These data were then compared with those obtained by the NSS in 2015 and 2016 for each Italian Region, in order to assess the completeness of reporting to the surveillance system.

\section{Methods}

\section{Data sources}

\section{Hospital discharge records}

All the hospitalizations made in public and private Italian hospitals are routinely collected in the HDR database, a systematic data collection on health care at national level, including both demographic and clinical information, such as primary and secondary diagnoses, primary and secondary diagnostic/therapeutic procedures, type of discharge, and hospital length of stay [5]. Diagnoses and procedures are coded using the "International Classification of Diseases, $9^{\text {th }}$ Revision, Clinical Modification" (ICD-9-CM).

We selected hospital discharges, among children aged less than 15 years, due to AFP based on the following ICD-9-CM codes as indicated in primary or secondary diagnoses [6]:

045.0X "Acute paralytic poliomyelitis specified as bulbar"

045.1X "Acute poliomyelitis with other paralysis"

357.0 "Acute infective polyneuritis" (approximates GBS)

359.9 "Myopathy, unspecified" (approximates Flaccid paralysis)

323.9 "Unspecified causes of encephalitis, myelitis, and encephalomyelitis" (approximates Acute transverse myelitis)

\section{The Italian National Surveillance System for AFP}

The active surveillance system was established at a national level in 1997, and it is coordinated by the ISS hosting the WHO collaborative centre for reference and research on polio. All AFP notifications included clinical and laboratory information and followed-up for residual paralysis at 60-90 days to ensure that the cases were not due to PV [4]. In particular, according to the WHOrecommended surveillance standard of poliomyelitis, the following criteria were considered:

clinical case definition: any child under 15 years of age with AFP or any person of any age with paralytic illness if polio is suspected;

case classification: suspected case (meets the clinical case definition) or confirmed case (presence of poliovirus in stool samples).

For all notified AFP to the RRC, stool samples were collected for laboratory confirmation. After 60 days from case notification, a follow-up questionnaire with more detailed information has to be sent to ISS and MoH to clarify the conclusive diagnosis. A first performance indicator is the number of AFP cases not due to polio per 100,000 children $<15$ years of age per year, which is indicative of the sensitivity of the surveillance system $(\geq 1 /$ $100,000)$. A second indicator is the proportion of AFP cases $(\geq 80 \%)$ for which stool specimens in "good" conditions were collected [7].

According to the national regulation, ethics approval was not required as clinical isolates were collected, 
processed, and stored as part of routine clinical care by the hospital laboratories participating in the network. The surveillance activities are required by the Global Polio Eradication Initiative (GPEI) of the WHO in the "Polio Eradication \& Endgame Strategic Plan 20132018", and by the Italian Ministry of Health (ref. 0004114-08/02/2018-DGPRE-DGPRE-P).

Data used for our study was not publically available.

\section{Statistical analysis}

All the HDRs between 1st of January 2005 and 31st of December 2016 were extracted from the $\mathrm{MoH}$ database. We considered the first hospital admission in case the same patient experienced more than one event, and then we excluded all hospitalization events occurring in the years 2005 and 2006, in order to exclude patients with sequelae from previous AFP discharges.

The number of hospitalizations, both overall and by specific diagnostic codes, and the AFP incidence rates were summarized by calendar year, gender, age groups, and geographical area (i.e., Northern, Central, and Southern Italy, based on the Italian National Institute of Statistics classification - ISTAT).

For the comparison between HDR and NSS data, we focused on the last 2 years of the considered period, 2015-2016, to study in-depth not only the differences between the two databases for each Italian Region, but also to highlight the discrepancies among Regions and how those vary in the latest data available year. Therefore, the absolute number and incidence rates (IR) of AFP coming from HDR data were calculated for each Region and compared with those reported by the NSS in the same period. Moreover, differences between the number of reported and expected cases notified to the NSS were calculated, to show the degree to which a Region was over or under the target value.

Statistical analysis was performed using the Stata software, version 13 (Stata Cooperation, College Station, Texas, USA).

\section{Results}

We identified 4163 hospital events with a diagnosis compatible with AFP between 2007 and 2016 in Italy. Most cases were clinically diagnosed as unspecified myopathy (1803, 43.3\%), followed by encephalitis/myelitis/ encephalomyelitis $(1408,33.8 \%)$, and acute infective polyneuritis $(956,23.0 \%)$ (Table 1). A decreasing trend was observed for all diagnoses during the study period, and overall the annual AFP incidence rate (IR) declined from 5.5 to 4.5 per 100,000 children. In addition, the proportion of the three main diagnoses out of the total AFP cases for each calendar year remained quite stable over time. It is of note that 3 "Acute paralytic poliomyelitis specified as bulbar" cases ( 2 cases 045.00 unspecified type poliovirus, 1 case 045.02 poliovirus type I), and 1 "Acute poliomyelitis with other paralysis" case (045.10 unspecified type poliovirus) were incorrectly coded as ascertained after an investigation of ISS and $\mathrm{MoH}$ on medical records. A single vaccine-associated event, occurred in an Albanian subject before his arrival in Italy in 2014, was recorded as "Acute poliomyelitis with other paralysis, poliovirus type III".

Table 2 shows the main characteristics of the AFP cases among children aged 0-14 years reported in HDR database in the last 2 years of the study period, 20152016. Females were less frequently affected by AFP as compared to males [incidence rate ratio (IRR) $0.7 / 100$, 000], and fewer cases of AFP were recorded among children aged 5-9 and 10-14 years compared to those aged $0-4$ years (IRR 0.6 and 0.5 per 100,000). In addition, small differences in the AFP incidence rates were found among the three geographic areas, as the Central and Southern Italy compared to Northern Italy (the most populated area, and with more hospital services in the paediatric setting) reported an IRR of 0.9 and 0.8 , respectively. Similar patterns of AFP types were observed in all geographic areas. The most common cause of AFP cases was myopathy (IR 1.9/100,000), followed by encephalitis/myelitis/encephalomyelitis (IR 1.4/100,000) and acute infective polyneuritis $(0.9 / 100,000)$.

The AFP cases from HDR were compared to those reported to the NSS in 2015-2016, analysing data from each of the 20 Italian Regions (Trentino-Alto Adige Region is formed by Bolzano and Trento Autonomous Provinces) (Table 3). Twelve cases notified to the NSS were not included in the analysis, because they did not fit with the considered ICD-9-CM specific codes. Major AFP IR (per 100,000 children) coming from HDR data were detected in Liguria (17.4), Sicily (5.7) and Veneto (5.1), whereas the lowest values were recorded in Umbria (0.4) and Molise (1.4) (Fig. 1a). Of note, the highest percentages of hospitalization among non-residents were recorded in Liguria and Lazio Regions, nearly 64 and $40 \%$ respectively, indicating an interregional mobility. Regarding the AFP notified to the NSS, the completeness of reporting varied by Regions. Considering the difference between the number of reported and expected cases (based on 1/100,000 rate), 11 out of 21 Regions failed to achieve the target (values $<0$ ), and the highest discrepancies were observed in the Northern Regions (Lombardy, Emilia-Romagna, Tuscany, and Piedmont) (Fig. 1b). Campania, Lazio and Calabria were over the expected value (difference $>0$ ), while for 7 Regions no difference was found.

Overall, the total number of AFP cases from NSS in Italy did not reach the expected value, which is nearly 83 cases considering an Italian population aged under 15 years of 8,300,000. In 2015-2016, AFP cases from NSS 
Table 1 Acute flaccid paralysis cases and incidence rates, Italy 2007-2016

\begin{tabular}{|c|c|c|c|c|c|c|c|c|c|c|c|c|}
\hline \multicolumn{3}{|c|}{ ICD-9-CM diagnosis codes } & \multicolumn{2}{|c|}{045.0} & \multicolumn{2}{|c|}{045.1} & \multicolumn{2}{|c|}{357.0} & \multicolumn{2}{|l|}{359.9} & \multicolumn{2}{|l|}{323.9} \\
\hline Year & AFP & $\mathbb{R}$ & N & $\%$ & N & $\%$ & N & $\%$ & N & $\%$ & N & $\%$ \\
\hline 2007 & 457 & 5.5 & 0 & 0.0 & 0 & 0.0 & 117 & 25.5 & 171 & 37.3 & 169 & 36.9 \\
\hline 2008 & 463 & 5.5 & 0 & 0.0 & 0 & 0.0 & 95 & 20.5 & 217 & 46.9 & 152 & 32.8 \\
\hline 2009 & 473 & 5.6 & 0 & 0.0 & 0 & 0.0 & 101 & 21.3 & 196 & 41.4 & 176 & 37.1 \\
\hline 2010 & 434 & 5.1 & 0 & 0.0 & 0 & 0.0 & 110 & 25.2 & 195 & 44.7 & 130 & 29.8 \\
\hline 2011 & 443 & 5.2 & 0 & 0.0 & 0 & 0.0 & 113 & 25.5 & 190 & 42.9 & 140 & 31.6 \\
\hline 2012 & 412 & 4.9 & 0 & 0.0 & 0 & 0.0 & 88 & 21.4 & 171 & 41.5 & 155 & 37.6 \\
\hline 2013 & 378 & 4.5 & 0 & 0.0 & 0 & 0.0 & 84 & 22.2 & 174 & 46.0 & 120 & 31.7 \\
\hline 2014 & 390 & 4.6 & 0 & 0.0 & 1 & 0.3 & 93 & 23.8 & 166 & 42.6 & 130 & 33.3 \\
\hline 2015 & 339 & 4.0 & 0 & 0.0 & 0 & 0.0 & 62 & 18.3 & 162 & 47.8 & 116 & 34.2 \\
\hline 2016 & 374 & 4.5 & 0 & 0.0 & 0 & 0.0 & 93 & 24.9 & 161 & 43.0 & 120 & 32.1 \\
\hline 2007-2016 & 4163 & 5.0 & 0 & 0.0 & 1 & 0.0 & 956 & 23.0 & 1803 & 43.3 & 1408 & 33.8 \\
\hline
\end{tabular}

Data were extracted from Hospital Discharge Records [5]

Italian population data were obtained from ISTAT database (www.demo.istat.it)

Five cases presented more than one diagnosis

ICD-9-CM diagnosis codes: 045.0 "Acute paralytic poliomyelitis specified as bulbar";

045.1 "Acute poliomyelitis with other paralysis"; 357.0 "Acute infective polyneuritis";

359.9 "Myopathy, unspecified"; 323.9 "Unspecified causes of encephalitis, myelitis, and encephalomyelitis"

357.0 code approximates Guillain-Barré Syndrome; 359.9 code approximates Flaccid paralysis; 323.9 code approximates Acute transverse myelitis

AFP acute flaccid paralysis, IR incidence rate $\times 100,000$

Table 2 Demographic and clinical characteristics of Acute Flaccid Paralysis cases, Italy 2015-2016

\begin{tabular}{|c|c|c|c|c|c|}
\hline AFP $=713$ & Number & Percent & $\mathbb{I R}$ & IRR & $95 \%$ CI IRR \\
\hline \multicolumn{6}{|l|}{ Gender } \\
\hline Male & 430 & 60.3 & 5.0 & - & \\
\hline Female & 283 & 39.7 & 3.5 & 0.7 & $0.6-0.8$ \\
\hline \multicolumn{6}{|l|}{ Class of age (years) } \\
\hline $0-4$ & 322 & 45.2 & 6.2 & - & \\
\hline $5-9$ & 198 & 27.8 & 3.5 & 0.6 & $0.5-0.7$ \\
\hline $10-14$ & 193 & 27.1 & 3.4 & 0.5 & $0.5-0.7$ \\
\hline \multicolumn{6}{|l|}{ Nationality } \\
\hline Italian & 627 & 87.9 & 4.2 & - & \\
\hline Other & 86 & 12.1 & 4.6 & 1.1 & $0.9-1.4$ \\
\hline \multicolumn{6}{|l|}{ Geographic area } \\
\hline North & 357 & 50.1 & 4.7 & - & \\
\hline Centre & 132 & 18.5 & 4.1 & 0.9 & $0.7-1.1$ \\
\hline South & 224 & 31.4 & 3.8 & 0.8 & $0.7-1.0$ \\
\hline \multicolumn{6}{|l|}{ Clinical characteristics } \\
\hline Acute infective polyneuritis (357.0) & 155 & 21.7 & 0.9 & - & \\
\hline Myopathy, unspecified (359.9) & 323 & 45.2 & 1.9 & 2.1 & $1.7-2.6$ \\
\hline Unspecified causes of encephalitis, myelitis, and encephalomyelitis (323.9) & 236 & 33.1 & 1.4 & 1.6 & $1.3-1.9$ \\
\hline
\end{tabular}

Data were extracted from Hospital Discharge Records [5]

Italian population data were obtained from ISTAT database (www.demo.istat.it)

357.0 code approximates Guillain-Barré Syndrome; 359.9 code approximates Flaccid paralysis; 323.9 code approximates Acute transverse myelitis

AFP acute flaccid paralysis, $I R$ incidence rate $\times 100,000$ population, $I R R$ incidence rate ratio, $C l$ confidence interval 
Table 3 Acute Flaccid Paralysis cases and incidence rates: comparison between Hospital Discharge Records and National Surveillance System, Italy 2015-2016

\begin{tabular}{|c|c|c|c|c|c|c|c|c|c|c|c|c|c|c|c|c|c|c|c|c|}
\hline \multirow[t]{2}{*}{ Regions } & \multicolumn{4}{|c|}{ AFP 2015} & \multicolumn{2}{|c|}{357.0} & \multicolumn{2}{|c|}{359.9} & \multicolumn{2}{|c|}{323.9} & \multicolumn{4}{|c|}{ AFP 2016} & \multicolumn{2}{|c|}{357.0} & \multicolumn{2}{|l|}{359.9} & \multicolumn{2}{|l|}{323.9} \\
\hline & $\mathrm{HDR}$ & HDR IR & NSS & NSS IR & $\mathrm{HDR}$ & NSS & $\mathrm{HDR}$ & NSS & $\mathrm{HDR}$ & NSS & $\mathrm{HDR}$ & HDR IR & NSS & NSS IR & $\mathrm{HDR}$ & NSS & $\mathrm{HDR}$ & NSS & $\mathrm{HDR}$ & NSS \\
\hline Piedmont & 19 & 3.3 & 3 & 0.5 & 4 & 3 & 7 & 0 & 8 & 0 & 27 & 4.8 & 5 & 0.9 & 3 & 2 & 11 & 2 & 13 & 1 \\
\hline Valle d'Aosta & 1 & 5.6 & 0 & 0.0 & 1 & 0 & 0 & 0 & 0 & 0 & 0 & 0.0 & 0 & 0.0 & 0 & 0 & 0 & 0 & 0 & 0 \\
\hline Lombardy & 60 & 4.2 & 4 & 0.3 & 10 & 2 & 28 & 0 & 22 & 2 & 57 & 4.0 & 14 & 1.0 & 15 & 8 & 25 & 0 & 17 & 6 \\
\hline A.P. Bolzano & 0 & 0.0 & 0 & 0.0 & 0 & 0 & 0 & 0 & 0 & 0 & 5 & 6.0 & 3 & 3.6 & 3 & 3 & 1 & 0 & 1 & 0 \\
\hline A.P. Trento & 1 & 1.2 & 1 & 1.2 & 1 & 1 & 0 & 0 & 0 & 0 & 3 & 3.8 & 1 & 1.3 & 1 & 1 & 1 & 0 & 1 & 0 \\
\hline Veneto & 33 & 4.8 & 3 & 0.4 & 4 & 3 & 16 & 0 & 13 & 0 & 37 & 5.4 & 10 & 1.5 & 10 & 9 & 12 & 0 & 15 & 1 \\
\hline Friuli-Venezia Giulia & 3 & 1.9 & 0 & 0.0 & 1 & 0 & 2 & 0 & 1 & 0 & 4 & 2.6 & 0 & 0.0 & 2 & 0 & 2 & 0 & 0 & 0 \\
\hline Liguria & 35 & 19.2 & 0 & 0.0 & 2 & 0 & 28 & 0 & 5 & 0 & 28 & 15.6 & 1 & 0.6 & 2 & 1 & 22 & 0 & 4 & 0 \\
\hline Emilia-Romagna & 13 & 2.2 & 4 & 0.7 & 4 & 2 & 5 & 0 & 4 & 2 & 31 & 5.2 & 1 & 0.2 & 11 & 0 & 10 & 0 & 10 & 1 \\
\hline Tuscany & 23 & 4.8 & 0 & 0.0 & 1 & 0 & 14 & 0 & 8 & 0 & 14 & 2.9 & 3 & 0.6 & 3 & 3 & 8 & 0 & 3 & 0 \\
\hline Umbria & 0 & 0.0 & 1 & 0.9 & 0 & 0 & 0 & 0 & 0 & 1 & 1 & 0.9 & 0 & 0.0 & 0 & 0 & 1 & 0 & 0 & 0 \\
\hline Marche & 8 & 3.9 & 1 & 0.5 & 3 & 0 & 3 & 0 & 2 & 1 & 10 & 5.0 & 1 & 0.5 & 3 & 0 & 6 & 0 & 1 & 1 \\
\hline Lazio & 41 & 5.0 & 2 & 0.2 & 10 & 2 & 26 & 0 & 5 & 0 & 35 & 4.3 & 5 & 0.6 & 10 & 3 & 19 & 0 & 6 & 2 \\
\hline Abruzzi & 6 & 3.5 & 2 & 1.2 & 1 & 1 & 1 & 0 & 4 & 1 & 7 & 4.1 & 0 & 0.0 & 1 & 0 & 2 & 0 & 4 & 0 \\
\hline Molise & 1 & 2.7 & 0 & 0.0 & 0 & 0 & 0 & 0 & 1 & 0 & 0 & 0.0 & 0 & 0.0 & 0 & 0 & 0 & 0 & 0 & 0 \\
\hline Campania & 16 & 1.8 & 7 & 0.8 & 6 & 4 & 7 & 0 & 3 & 3 & 20 & 2.2 & 0 & 0.0 & 5 & 0 & 10 & 0 & 5 & 0 \\
\hline Apulia & 22 & 3.8 & 5 & 0.9 & 2 & 5 & 7 & 0 & 13 & 0 & 27 & 4.8 & 8 & 1.4 & 9 & 3 & 6 & 0 & 11 & 5 \\
\hline Basilicata & 2 & 2.7 & 0 & 0.0 & 0 & 0 & 1 & 0 & 1 & 0 & 1 & 1.4 & 0 & 0.0 & 0 & 0 & 0 & 0 & 1 & 0 \\
\hline Calabria & 7 & 2.6 & 1 & 0.4 & 3 & 1 & 2 & 0 & 2 & 0 & 13 & 4.8 & 0 & 0.0 & 4 & 0 & 3 & 0 & 6 & 0 \\
\hline Sicily & 39 & 5.3 & 5 & 0.7 & 6 & 3 & 13 & 0 & 20 & 2 & 45 & 6.2 & 7 & 1.0 & 7 & 5 & 19 & 1 & 19 & 1 \\
\hline Sardinia & 9 & 4.5 & 1 & 0.5 & 3 & 1 & 2 & 0 & 4 & 0 & 10 & 5.1 & 1 & 0.5 & 4 & 0 & 3 & 0 & 3 & 1 \\
\hline Italy & 339 & 4.0 & 40 & 0.5 & 62 & 28 & 162 & 0 & 116 & 12 & 375 & 4.5 & 60 & 0.7 & 93 & 38 & 161 & 3 & 120 & 19 \\
\hline
\end{tabular}

Data were extracted from Hospital Discharge Records; Italian population data were obtained from ISTAT database (www.demo.istat.it)

AFP acute flaccid paralysis, HDR Hospital Discharge Records [5], NSS National Surveillance System [4], IR Incidence rate. ICD-9-CM diagnosis codes: 357.0 "Acute infective polyneuritis"; 359.9 "Myopathy, unspecified"; 323.9 "Unspecified causes of encephalitis, myelitis, and encephalomyelitis"

were $14 \%$ of those obtained by HDR data, indicating a high level of underreporting at a national level. Specifically, only $42.6 \%$ of acute infective polyneuritis cases, $13.1 \%$ of encephalitis/myelitis/encephalomyelitis cases, and $0.9 \%$ of myopathy cases reported in HDR were notified to the NSS.

\section{Discussion}

The present study aimed to evaluate the AFP surveillance system over a 10-year period in Italy by comparing data obtained from HDR with those from NSS in the last 2 years by each Italian region. The comparison indicates that NSS data are affected by underreporting, since the AFP cases from NSS were only $14 \%$ of those recorded in HDR during the years 2015-2016. Specifically, the percentage of notified cases differs by clinical diagnosis being almost absent for unspecified myopathies (359.9 ICD-9-CM code), and very low for encephalitis/myelitis/ encephalomyelitis with unspecified causes (323.9 ICD-9CM code), while GBS cases (included in 357.0 ICD-9$\mathrm{CM}$ code) have been notified. Even the underreporting exists, the NSS still plays a crucial role in identifying AFP cases that would remain undetected due to the disadvantage of HDRs, since they have the limitation that is not based on specific case definitions, and therefore not extremely accurate. This is demonstrated by coding errors for the discharge diagnoses of four not confirmed cases of poliomyelitis, lacking the requirement for a laboratory-based confirmation.

Although poliomyelitis has historically been responsible for most cases of flaccid paralysis infectionrelated, non-polio enteroviruses were well known to be associated with AFP/Acute Flaccid Myelitis (AFM) [8]. In particular, enterovirus D68 (EV-D68) has become increasingly recognized in the context of enhanced surveillance for polio-like diseases [9]. Large outbreaks of EV-D68 occurred in the United States, Canada, and Europe in 2014 and 2016, temporally and geographically associated with an increase in AFM [10]. During 2018, the United Kingdom described an increase in reports of AFP cases, with many cases diagnosed as the AFM occurred mainly among preschool children [11]. 
a)

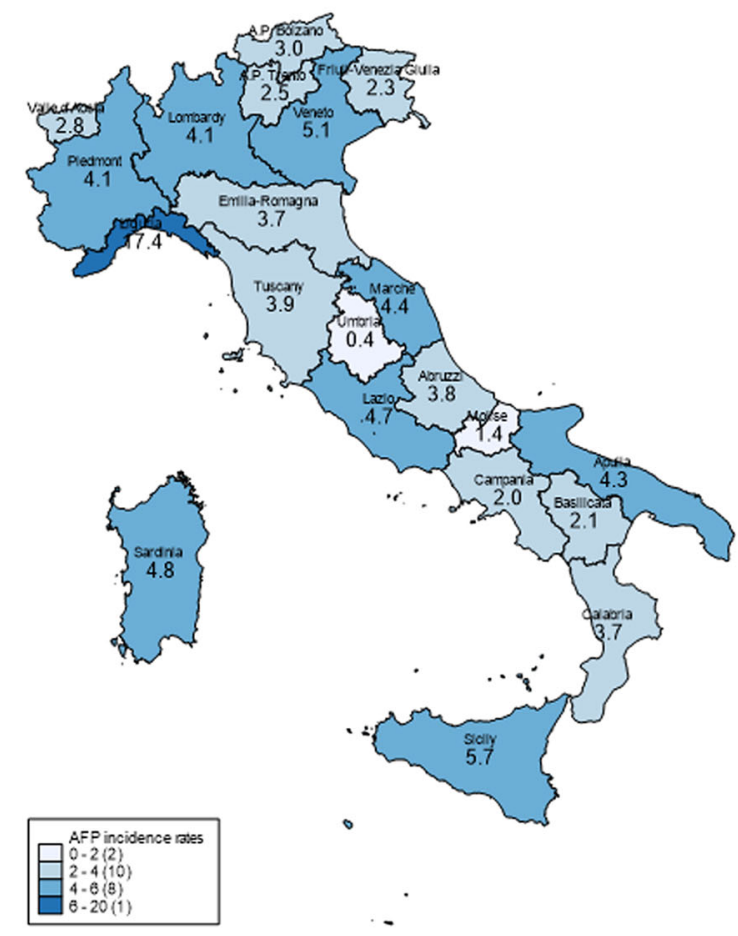

b)

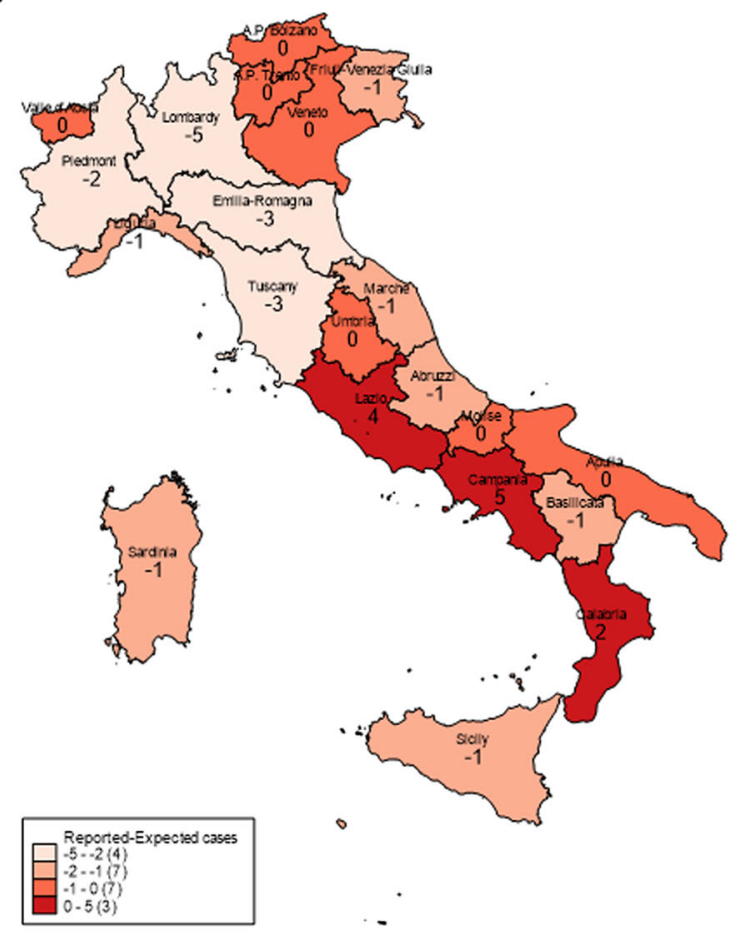

Fig. 1 a-b Acute Flaccid Paralysis by Italian Regions, years 2015-2016. Incidence rates per 100,000 children, data from Hospital Discharge Records (a); Difference between the number of reported and expected cases, data from the National Surveillance System (b). The maps have been produced using Stata software, version 13 (Stata Cooperation, College Station, Texas, USA)

In Italy, data on EV-D68 circulation are not systematically collected in the context of AFP surveillance.

In Italy, the last case of indigenous poliomyelitis due to a wild poliovirus occurred in 1982, while in 1988 the last imported case of polio infection was reported. Polio vaccination became mandatory in 1966 for children within the first year of age; in 2002, the inactivated Salk polio vaccine (IPV) completely replaced the Sabin oral polio vaccine (OPV) for safety reasons and risk-benefit considerations. The current National Vaccination Plan for 2017-2019 includes a primary vaccination with three doses at 3,5 , and 11 months of age and booster doses at 6 and 12-18 years of age [12]. Currently, in the EU area, Bosnia and Herzegovina, Romania and Ukraine are considered countries with a high risk of polio outbreak following the importation of wild PV or emergence of circulating vaccinederived PV due to suboptimal vaccination program performance and low immunity of the population [7]. Furthermore, several middle-income countries were considered at intermediate risk of polio transmission, as vaccination coverage appears to be declining and the quality of poliovirus surveillance is not optimal [13]. Moreover, in the three remaining endemic countries, Afghanistan, Pakistan and Nigeria, poliovirus transmission continued in 2018 [14].
In this scenario, maintaining a high vaccination coverage against polio minimizes the risk of spreading the disease in case of importation, and a highly sensitive surveillance system for AFP is essential to monitor polio-free status certification, and to respond to vaccinederived polioviruses outbreaks, which could circulate for several years after ending the use of OPV.

As established by the $\mathrm{WHO}$, at least 1 case of nonpolio AFP should be detected annually per 100,000 population under 15 years of age, which is an adequate quality of the surveillance system [1]. However, since the beginning of the NSS, the average value of the non-polio AFP rate was 0.7 in Italy and reached the target level only in 2003. Of note, this value is in line with the average AFP detection rate $(0.6 / 100,000)$ from 1998 to 2015 in Spain [15]. In this country, the quality of AFP surveillance has decreased over time, particularly after the switch from OPV to IPV, reflecting the loss of awareness about polio, when it is very unlikely that cases of paralysis produced by wild PV or associated with the vaccine-derived PV will occur. However, notifications and hospital admissions with a clinical diagnosis compatible with AFP have followed the same pattern, indicating that, despite the underreporting, the surveillance works relatively well. Overall, in regions certified as polio-free for many years (the Americas in 1994, 
Western Pacific Region in 2000, and WHO European Region in 2002 [2]), the sensitivity and quality of AFP reports decreased over time [16].

In our country, several possible reasons have been identified that contribute to the underreporting in AFP surveillance system: the long absence of polio in Italy with consequently loss of interest in preventive issues and maintainance of polio-free status; the lack of knowledge and awareness of clinicians to associate the surveillance with non-polio AFP; the presence of a confirmed alternative diagnosis. Specifically, some paediatricians could equate AFP only to acute poliomyelitis and not with a wide range of conditions that AFP can present, such as making a specific diagnosis of GBS rather than labelling a case as AFP. Indeed, the widespread eradication of poliomyelitis has led to GBS becoming the most frequent cause of acute and sub-acute flaccid paralysis. Furthermore, the scarce notification of AFP cases could be related to the physicians' lack of knowledge of the surveillance criteria and of the importance of reporting and investigating all AFP cases, or finally could be due to management problems that are much more evident in some parts of Italy. Overall, several efforts could be made to enhance and strengthen the surveillance activities, by increasing awareness of optimal investigation and management of cases, as well as specific training to increase knowledge among clinicians, microbiologists, public health specialists and other key partners involved in notifiable disease reporting. In addition, laboratory testing for non-polio enteroviruses could be implemented both to monitor polio eradication and to establish sensitive surveillance to detect other enteroviruses that cause severe symptoms such as EV-D68.

\section{Conclusions}

Our study shows that the Italian AFP surveillance system is affected by underreporting and that the completeness of reporting varies by Italian Regions. Therefore, it is important to closely monitor the activity of the various paediatric centres in the country to improve the quality of the surveillance system. Furthermore, the regular adoption of the cross-evaluation of notified data, also through the review of HDRs, could help to plan effective actions for the improvement of the NSS.

\section{Abbreviations \\ AFP: Acute flaccid paralysis; GBS: Guillain-barré syndrome; HDR: Hospital discharge records; ICD-9-CM: International Classification of Diseases, 9th Revision, Clinical Modification; IPV: Inactivated salk polio vaccine; IR: Incidence rate; ISS: Istituto Superiore di Sanità; MoH: Ministry of Health; NSS: National Surveillance System; OPV: Oral polio vaccine; PV: Poliovirus; RRC: Regional Reference Centres; WHO: World Health Organization}

\section{Acknowledgements}

The authors thank Alessandra D'Alberto, Department of Prevention of Communicable Diseases and International Prophylaxis of the Italian Ministry of Health.
\$Regional Reference Centres of the National Surveillance System for Acute flaccid paralysis: Filippo Ansaldi - Dipartimento di Scienze della Salute,Università degli Studi di Genova; Sandro Binda, Laura Pellegrinelli Dipartimento di Scienze Biomediche per la Salute, Università degli Studi di Milano;Guglielmo Bonaccorsi, Chiara Lorini - Dipartimento di Scienze della Salute, Università degli Studi di Firenze; Silvio Brusaferro - Dipartimento di AreaMedica dell'Università degli studi di Udine; Barbara Camilloni Dipartimento di Medicina, Università degli Studi di Perugia; Benita Capannolo, CristianaMancini - Dipartimento di prevenzione ASL 1 Abruzzo, L'Aquila; Valter Carraro - Azienda Provinciale per i Servizi Sanitari, Dipartimento di Prevenzione,Trento; Paolo Castiglia, Antonella Arghittu Dipartimento di Scienze Mediche, Chirurgiche e Sperimentali, Azienda Ospedaliero Universitaria- Universitàdegli Studi di Sassari; Marcello Mario D'Errico - Dipartimento di Scienze Biomediche e Sanità Pubblica, Università Politecnica delle Marche, Ancona;Carlo De Stefano - Ospedale San Carlo, Potenza; Alfredo Focà - Dipartimento di Scienze Mediche, Università Magna Grecia di Catanzaro; CinziaGerminario, Angela Larocca - Dipartimento di Scienze Biomediche e Oncologia Umana, Università degli Studi di Bari Aldo Moro; Giovanni M.Giammanco, Simona De Grazia - Dipartimento Promozione della Salute, Materno-Infantile, di Medicina Interna e Specialistica di Eccellenza, Universitàdegli Studi di Palermo; Guido Maria Grasso Dipartimento di Medicina e Scienze della Salute, Università degli Studi del Molise, Campobasso; DanielaLombardi - SeREMI ASL AL, Alessandria; Francesca Russo, Giuseppina Napoletano, Francesca Zanella - Direzione Prevenzione, Sicurezza Alimentare,Veterinaria della Regione Veneto, Venezia; Silvia Spertini - Azienda Sanitaria dell'Alto Adige, Dipartimento di Prevenzione, Bolzano; Licia Veronesi,Paola Affanni - Dipartimento di Medicina e Chirurgia, Università di Parma; Maria Triassi, Francesca Pennino -

Dipartimento di Sanità Pubblica, Universitàdegli Studi Federico II, Napoli; Francesco Vairo - Servizio regionale per la sorveglianza e il controllo delle malattie infettive (SERESMI) - Regione Lazio,Istituto nazionale per le malattie infettive Lazzaro Spallanzani, Roma

\section{Authors' contributions}

Regional Reference Centres of the National Surveillance System for Acute flaccid paralysis. PS conceived the study and wrote the manuscript. SB performed the statistical analysis and wrote the manuscript. SFi, SF and CA participated actively in the AFP surveillance together with GB. All authors read and approved the manuscript.

\section{Funding}

This work was partially supported by a grant from the Italian Ministry of Health, "National surveillance of the acute flaccid paralysis and research of poliovirus and other enteroviruses in immunodeficient patients and in the environment", 2015-2016, for the laboratory activities within the surveillance of AFP in Italy. The design of the present study, analysis, and interpretation of data were performed by the ISS, Department of Infectious Diseases as Reference National Laboratory for AFP. The surveillance system was included in the Decree of the President of the Council of Ministers, 3rd March 2017.

\section{Availability of data and materials}

Data supporting the results reported in the article include the Hospital discharge records and the National surveillance system database. HDR data are sent by hospitals to the Regional Health Authorities that transmit the database to the Ministry of Health. The database is available at the Istituto Superiore di Sanità as agreed with the "Collaboration agreement between the Ministry of Health and ISS for the use of Hospital discharge information flow to conduct studies in Public Health". Regarding the AFP National Surveillance System database, all records are shared with the Italian Ministry of Health, as the competent body for the AFP surveillance in Italy. Data are available from the authors upon reasonable request and with permission of the Italian Ministry of Health.

\section{Ethics approval and consent to participate}

Ethics approval was not required as clinical isolates were collected, processed, and stored as part of routine clinical care by the collaborating hospital laboratories participating in the network. As our study did not involve interventions or additional clinical samples to be collected, no consent to participate was required. The surveillance activities are within the Global Polio Eradication Initiative (GPEI) of the WHO in the "Polio Eradication 
\& Endgame Strategic Plan 2013-2018", and by the Italian Ministry of Health (ref. 0004114-08/02/2018-DGPRE-DGPRE-P).

\section{Consent for publication}

Not applicable. Our manuscript does not contain any individual person's data in any form, including any individual details, images or videos.

\section{Competing interests}

The authors declare that they have no competing interests.

Received: 12 March 2019 Accepted: 12 September 2019

Published online: 15 November 2019

\section{References}

1. WHO. Acute Flaccid Paralysis Surveillance: the surveillance strategy for poliomyelitis eradication. Wkly Epidemiol Rec. 1998;16:113-20.

2. Smith J, Leke R, Adams A, Tangermann RH. Certification of polio eradication: process and lessons learned. Bull World Health Organ. 2004;82:24-30.

3. Wilder-Smith A, Leong WY, Fernandez Lopez L, Amaku M, Quam M, Khan K, et al. Potential for international spread of wild poliovirus via travelers. BMC Med. 2015;13:133.

4. Istituto Superiore di Sanità [Italian National Institute of Health]. Scheda di segnalazione per la sorveglianza della paralisi flaccida acuta [Reporting sheet of the acute flaccid paralysis surveillance. Available from: http://www. salute.gov.it/imgs/C_17_pagineAree_820_listaFile_itemName_4_file.pdf. Accessed 24 Oct 2019

5. La scheda di dimissione ospedaliera [Hospital discharge records]. Available from: http://www.salute.gov.it/portale/temi/p2_6.jsp?lingua=italiano\&id= 1232\&area=ricoveriOspedalieri\&menu=vuoto. Accessed 24 Oct 2019.

6. D'souza RM. Retrospective hospital-based searches for cases of acute flaccid paralysis. Aust N Z J Public Health. 2002;26(1):45-9.

7. Snider CJ, Diop OM, Burns CC, Tangermann RH, Wassilak SGF. Surveillance systems to track progress toward polio eradication—worldwide, 2014-2015. Morb Mortal Wkly Rep. 2016;65:346-51.

8. Bitnun A, Yeh EA. Acute flaccid paralysis and Enteroviral infections. Curr Infect Dis Rep. 2018;20(9):34.

9. Tokarz R, Firth C, Madhi SA, Howie SR, Wu W, Sall AA, et al. Worldwide emergence of multiple clades of enterovirus 68. J Gen Virol. 2012;93:1952-8.

10. Dyda A, Stelzer-Braid S, Adam D, Chughtai AA, MacIntyre CR. The association between acute flaccid myelitis (AFM) and Enterovirus D68 (EVD68) - what is the evidence for causation?. Euro Surveill. 2018;23(3). Available at: https://www.ncbi.nlm.nih.gov/pubmed/29386095.

11. The United Kingdom Acute Flaccid Paralysis Afp Task Force. An increase in reports of acute flaccid paralysis (AFP) in the United Kingdom, 1 January 2018-21 January 2019: early findings. Euro Surveill. 2019;24(6):1900093. Available at: https://www.ncbi.nlm.nih.gov/pmc/articles/PMC6373064/.

12. Piano nazionale Prevenzione vaccinale 2017-2019 [National Vaccination Plan 2017-2019]. Available from: http://www.salute.gov.t//imgs/C_17 pubblicazioni_2571_allegato.pdf. Accessed 24 Oct 2019.

13. World Health Organization Regional Office for Europe. 32nd Meeting of the European Regional Commission for Certification of Poliomyelitis Eradication. Report 2018. Available from: http://www.euro.who.int/en/health-topics/ communicable-diseases/poliomyelitis/publications/2018/32nd-meeting-ofthe-european-regional-commission-for-certification-of-poliomyelitiseradication-rcc-report-2018. Accessed 24 Oct 2019.

14. WHO. Review of polio endemic countries. 2018. Available from: https:// www.who.int/immunization/sage/meetings/2018/october/4_Review_Polio_ Endemic_Countires_Report_IMB_Final_revised.pdf?ua=1.

15. Masa-Calles J, Torner N, López-Perea N de Viarce Torres de Mier M Fernández-Martínez B, Cabrerizo M et al. Acute flaccid paralysis (AFP) surveillance: challenges and opportunities from 18 years' experience, Spain, 1998 to 2015. Euro Surveill. 2018;23(47). Available at: https://www. eurosurveillance.org/content/10.2807/1560-7917.ES.2018.23.47.1700423.

16. Wassilak SGF, Williams CL, Murrill CS, Dahl BA, Ohuabunwo C, Tangermann RH. Using Acute Flaccid Paralysis Surveillance as a Platform for VaccinePreventable Disease Surveillance. J Infect Dis. 2017;216(suppl_1):S293-8.

\section{Publisher's Note}

Springer Nature remains neutral with regard to jurisdictional claims in published maps and institutional affiliations.

Ready to submit your research? Choose BMC and benefit from:

- fast, convenient online submission

- thorough peer review by experienced researchers in your field

- rapid publication on acceptance

- support for research data, including large and complex data types

- gold Open Access which fosters wider collaboration and increased citations

- maximum visibility for your research: over $100 \mathrm{M}$ website views per year

At BMC, research is always in progress.

Learn more biomedcentral.com/submissions 